\title{
Metabolic profiling of elite athletes with different cardiovascular demand
}

\author{
Fatima Al-Khelaifi ${ }^{1,2}$ ｜ Francesco Donati $^{3}$ ｜ Francesco Botrè $^{3}$ | Aishah Latiff ${ }^{1}$ | \\ David Abraham $^{2}$ | Aroon Hingorani ${ }^{4}$ | Costas Georgakopoulos ${ }^{1}$ | Karsten Suhre \\ Noha A. Yousri ${ }^{6,7}$ | Mohamed A. Elrayess ${ }^{1,2,8}$ iD
}

\author{
${ }^{1}$ Anti Doping Laboratory Qatar, Doha, \\ Qatar \\ ${ }^{2}$ Royal Free Campus, UCL-Medical School, \\ London, UK \\ ${ }^{3}$ Laboratorio Antidoping, Federazione \\ Medico Sportiva Italiana, Rome, Italy \\ ${ }^{4}$ UCL Institute of Cardiovascular Science, \\ University College London, London, UK \\ ${ }^{5}$ Department of Physiology and \\ Biophysics, Weill Cornell Medical College \\ in Qatar, Qatar-Foundation, Doha, Qatar \\ ${ }^{6}$ Computers and System \\ Engineering, Alexandria University, \\ Alexandria, Egypt \\ ${ }^{7}$ Department of Genetic Medicine, Weill \\ Cornell Medical College in Qatar, Qatar- \\ Foundation, Doha, Qatar \\ ${ }^{8}$ Biomedical Research Center, Qatar \\ University, Doha, Qatar

\section{Correspondence} \\ Mohamed A. Elrayess, Anti Doping \\ Laboratory Qatar, Sports City, P.O Box \\ 27775, Doha, Qatar. \\ Email: melrayess@adlqatar.qa \\ Funding information \\ Qatar National Research Fund, Grant/ \\ Award Number: NPRP7-272-1-041
}

\begin{abstract}
Intensive exercise of elite athletes can lead to physiological alterations in the cardiovascular system in response to increased stroke volume and blood pressure, known collectively as cardiovascular demand (CD). This study aimed to compare metabolic differences in elite athletes with high vs low/moderate CD and to reveal their underlying metabolic pathways as potential biomarker signatures for assessing health, performance, and recovery of elite athletes. Metabolic profiling of serum samples from 495 elite athletes from different sport disciplines (118 high CD and 377 low/moderate $\mathrm{CD}$ athletes) was conducted using non-targeted metabolomics-based mass spectroscopy combined with ultra-high-performance liquid chromatography. Results show that DAGs containing arachidonic were enriched in high CD together with branched-chain amino acids, plasminogens, phosphatidylcholines, and phosphatidylethanolamines, potentially indicating increased risk of cardiovascular disease in the high CD group. Gamma-glutamyl amino acids and glutathione metabolism were increased in low/moderate CD group, suggesting more efficient oxidative stress scavenging mechanisms than the high CD group. This first most comprehensive metabolic profiling of elite athletes provides an evidence that athletes with different CD show a unique metabolic signature that reflects energy generation and oxidative stress and potentially places the high $\mathrm{CD}$ group at a higher risk of cardiovascular disease. Further studies are warranted for confirmation and validation of findings in other sport groups in light of potential confounders related to limited available information about participants.
\end{abstract}

\section{K E Y W O R D S}

cardiovascular demand, cardiovascular disease, elite athletes, metabolomics

\section{1 | INTRODUCTION}

Athletes competing in national or international sport events are considered elite athletes. ${ }^{1}$ Their intensive physical training is associated with electrical, structural, and functional myocardial adaptations that help improve their athletic performance. ${ }^{2,3}$ The type and intensity of their respective sport disciplines determine the metabolic demands for their local and systemic blood flow, whereas the duration of training causes regulatory disturbances and constraints in their

Yousri and Elrayess equally contributed to this study. 
cardiovascular function over time. ${ }^{4}$ In response to aerobic exercise, for example, the cardiovascular system can reach up to $80 \%$ of maximum cardiac output in order to meet the need to deliver oxygen to the exercising body organs while regulating the mean arterial pressure.,

Information related to peak static (maximal voluntary contraction, MVC) and dynamic (maximal oxygen uptake, MaxO2) components achieved during competition for athletes from different sporting disciplines are used to classify them into various classes of cardiovascular demand (CD). ${ }^{7}$ The increasing dynamic component results in a greater cardiac output, whereas the increasing static component results in an elevated blood pressure load. Accordingly, total cardiovascular demand that comprises both cardiac output and blood pressure is used to classify sports into five sport groups that range from low static/low dynamic (such as golf and riflery) to high static/high dynamic (such as boxing and cycling). ${ }^{7}$ Despite multiple physiological studies describing cardiac output and blood pressure of different groups of athletes ${ }^{8}$ a more comprehensive monitoring of blood metabolic biomarkers may provide valuable insight into the physiological and pathophysiological alterations underlying athletes long-term performance and health.

Metabolomics presents a comprehensive approach for detecting metabolic changes in response to dietary, lifestyle, and environmental factors, ${ }^{9}$ including profiles associated with performance, fatigue, and health issues. ${ }^{10,11}$ Previous non-targeted metabolomics studies revealed metabolic alterations in response to exercise, ${ }^{10,12,13}$ including changes in glucose, lipid, amino acid, and energy metabolites. ${ }^{10,13}$ Metabolic changes were observed in adenosine triphosphate (ATP) synthesis and beta-oxidation of fatty acids ${ }^{12}$ as well as elevation in plasma lactate $\mathrm{e}^{14,15}$ and adenine breakdown products. ${ }^{16}$ Components of tricarboxylic acid (TCA) cycle were also changed in response to aerobic energy production in skeletal muscle biopsies. ${ }^{17,18}$

Despite multiple studies focusing on the impact of exercise on athletes' metabolomics, profiling of metabolic changes in elite athletes from various sport disciplines characterized by different $\mathrm{CD}$ would provide deeper insight into their physiological state and cardiovascular adaptation in response to their respective sports. ${ }^{11}$ Measurement of these alterations would offer valuable information of the physical status of elite athletes and their adaptation to exercise. This could intern help in managing their future training programs and avoiding potential disorders related to excessive training as well as improving their performance in general. We have recently shown in a pilot study of 191 elite athletes that those engaged in high-power and high-endurance sports show a distinctive metabolic signature associated with steroid biosynthesis, fatty acid metabolism, oxidative stress, and energy-related metabolites. ${ }^{19}$ In this study, we aim to extend our investigation to confirm known and identify novel metabolites associated with $\mathrm{CD}$ as a potential biomarkers of athletes' health, performance, and recovery from injury in a new cohort of 495 elite athlete belonging to different sport disciplines.

\section{2 | MATERIALS AND METHODS}

\section{1 | Study design}

Four hundred ninety-five elite athletes from different sport disciplines, who participated in national or international

\begin{tabular}{|c|c|c|c|}
\hline & & \multicolumn{2}{|l|}{ Endurance } \\
\hline & & $\begin{array}{l}\text { Moderate }(40 \%-70 \% \\
\text { VO2max) }\end{array}$ & High (>70\% VO2max) \\
\hline \multirow[t]{3}{*}{ Power } & $\begin{array}{l}\text { High } \\
(>50 \% \mathrm{MVC})\end{array}$ & $\begin{array}{l}\text { Wrestling }(3 \mathrm{M}) \text {, Judo } \\
(3 \mathrm{M})\end{array}$ & $\begin{array}{l}\text { Boxing }(1 \mathrm{M} / 16 \mathrm{~F}) \text {, } \\
\text { Heptathlon }(1 \mathrm{M}), \\
\text { Rowing }(6 \mathrm{M} / 7 \mathrm{~F}), \\
\text { Cycling }(31 \mathrm{M} / 4 \mathrm{~F})\end{array}$ \\
\hline & $\begin{array}{l}\text { Moderate } \\
(20 \%-50 \% \text { MVC })\end{array}$ & $\begin{array}{l}\text { Athletics }(15 \mathrm{M} / 22 \mathrm{~F}) \text {, } \\
\text { Rugby }(16 \mathrm{M}), \text { Triple } \\
\text { Jump }(1 \mathrm{M})\end{array}$ & $\begin{array}{l}\text { Athletics 200-800 m } \\
(4 \mathrm{M}), \text { Hockey }(1 \mathrm{~F}), \\
\text { Skiing Cross Country } \\
(1 \mathrm{M}), \text { Basketball }(3 \mathrm{M}) \text {, } \\
\text { Swimming }(22 \mathrm{M} / 16 \mathrm{~F})\end{array}$ \\
\hline & $\begin{array}{l}\text { Low } \\
(<20 \% \mathrm{MVC})\end{array}$ & $\begin{array}{l}\text { Baseball }(2 \mathrm{M}) \text {, Volleyball } \\
(1 \mathrm{M})\end{array}$ & $\begin{array}{l}\text { Tennis }(1 \mathrm{M} / 1 \mathrm{~F}), \text { Soccer } \\
(315 \mathrm{M}) \text {, Athletics } \\
1500-3000 \mathrm{~m}(3 \mathrm{M})\end{array}$ \\
\hline
\end{tabular}

Categorization of elite athletes in groups based on sport disciplines characterized by variable peak dynamic (maximal oxygen uptake percentage; $\mathrm{VO} 2$ ) and peak static (maximal voluntary muscle contraction percentage; $\mathrm{MVC}$ ) components reached during competition as described previously. ${ }^{7}$ The number and gender ( $\mathrm{M}$ for males and $\mathrm{F}$ for females) of participants per group are also shown. Sport types were further categorized into two classes based on CD (high in gray and low in white) that were used in the statistical analysis.
TA B LE 1 Classification of study participants 
sport events and tested negative for doping substances at anti-doping laboratories in Qatar and Italy, were included in this study in accordance with the World Medical Association Declaration of Helsinki. Informed consents were obtained from all participants. All protocols were approved by the Institutional Research Board of anti-doping lab Qatar (F2014000009). Serum samples, collected randomly by the doping control officers for anti-doping human growth hormone tests, were used for metabolomics studies as described previously. ${ }^{19}$ Accordingly, only information related to sport group (shown in Table 1), ethnicity (85\% Europeans, 10\% Americans and 5\% Africans), and gender (87\% males, 13\% females) were available to researchers. Total CD was used to classify sports into two groups (low/moderate CD [ $\mathrm{n}=377$ ], shown in white in Table 1, vs high CD $(\mathrm{n}=118)$, shown in gray in Table 1. There was no evidence of population stratification in $\mathrm{CD}$ groups based on athletes' ethnicities.

\section{2 $\mid$ Metabolomics}

Profiling of metabolites was achieved using validated protocols at Metabolon, Durham, NC, USA, using Waters ACQUITY ultra-high-performance liquid chromatography (UPLC) and a Thermo Scientific Q-Exactive high-resolution/ accurate mass spectrometer interfaced with a heated electrospray ionization (HESI-II) source and Orbitrap mass analyzer operated at 35000 mass resolution.

\section{3 | Sample preparation}

Upon delivery, sample inventories were confirmed and samples were stored at $-80^{\circ} \mathrm{C}$ until time of processing. Sample preparation was performed using automated MicroLab STAR ${ }^{\circledR}$ system (Hamilton Company). Recovery standards were added prior to the first step in the extraction process for QC purposes. Proteins were precipitated in methanol under vigorous shaking for 2 minutes (Glen Mills GenoGrinder 2000), followed by centrifugation to remove proteins, dissociate small molecules that are either bound to proteins or trapped in the precipitated protein matrix, and to recover chemically diverse metabolites. The resulting extract was divided into five fractions as follows: two for analysis by two separate reverse phase (RP)/ Ultra-high-performance liquid chromatography-tandem mass spectroscopy (UPLC-MS/MS) methods with positive ion mode electrospray ionization (ESI; for detection of positive early and positive late fractions), one for analysis by RP/UPLC-MS/MS with negative ion mode ESI, one for analysis by HILIC/UPLC-MS/MS with negative ion mode ESI (for the detection of the polar compounds), and one sample was reserved for backup. Samples were placed briefly on a TurboVap ${ }^{\circledR}$ (Zymark) to remove the organic solvent. The sample extracts were stored overnight under nitrogen before preparation for analysis.

\section{4 | Quality control}

Several controls were analyzed together with the tested samples. These included a pooled matrix generated by taking a small volume of each experimental sample served as a technical replicate throughout the data set. Extracted water samples were used as blanks. Additionally, a cocktail of QC standards that were carefully chosen not to interfere with the measurement of endogenous compounds was spiked into every analyzed sample, allowing instrument performance monitoring and aiding chromatographic alignment. Instrument variability was determined by calculating the median relative standard deviation (RSD) for the standards that were added to each sample prior to injection into the mass spectrometers. Overall process variability was determined by calculating the median RSD for all endogenous metabolites (ie, non-instrument standards) present in $100 \%$ of the pooled matrix samples. Experimental samples were randomized across the platform and run with QC samples that were spaced evenly among the injections.

\section{5 | Ultra-high-performance liquid chromatography-tandem mass spectroscopy}

The dried sample extract was reconstituted in solvents specific to each of the four methods. Each reconstitution solvent contained a series of standards at fixed concentrations to ensure injection and chromatographic consistency. One aliquot was analyzed using acidic positive ion conditions, chromatographically optimized for more hydrophilic compounds. In this method, the extract was gradient eluted from a C18 column (Waters UPLC BEH C18-2.1 × $100 \mathrm{~mm}, 1.7 \mu \mathrm{m}$ ) using water and methanol, containing $0.05 \%$ perfluoropentanoic acid (PFPA) and $0.1 \%$ formic acid (FA). Another aliquot was also analyzed using acidic positive ion conditions; however, it was chromatographically optimized for more hydrophobic compounds. In this method, the extract was gradient eluted from the same mentioned C18 column using methanol, acetonitrile, water, $0.05 \%$ PFPA, and $0.01 \%$ FA and was operated at an overall higher organic content. Another aliquot was analyzed using basic negative ion optimized conditions using a separate dedicated $\mathrm{C} 18$ column.The basic extracts were gradient eluted from the column using methanol and water, however, with $6.5 \mathrm{mM}$ ammonium bicarbonate at $\mathrm{pH}$ 8. The fourth aliquot was analyzed via negative ionization following elution from a HILIC column (Waters UPLC BEH Amide $2.1 \times 150 \mathrm{~mm}, 1.7 \mu \mathrm{m}$ ) using a gradient consisting of water and acetonitrile with $10 \mathrm{mM}$ ammonium formate, $\mathrm{pH}$ 10.8. The MS analysis alternated between MS and data-dependent MS/MS scans using dynamic exclusion. The scan range varied slighted between methods but covered $70-1000 \mathrm{~m} / \mathrm{z}$. Raw data files were archived and extracted as described below. 


\section{6 | Data extraction and compound identification}

Raw data were extracted, peak-identified, and QC processed using Metabolon's hardware and software. These systems are built on a web-service platform utilizing Microsoft's NET technologies, which run on high-performance application servers and fiber-channel storage arrays in clusters to provide active failover and load-balancing. Compounds were identified by comparison with library entries of purified standards or recurrent unknown entities. Metabolon maintains a library based on authenticated standards that contains the retention time/index (RI), mass to charge ratio $(\mathrm{m} / \mathrm{z})$, and chromatographic data (including MS/MS spectral data) on all molecules present in the library. Furthermore, biochemical identifications are based on the following three criteria: the correct retention time/index to the authentic standard, the correct $\mathrm{m} / \mathrm{z}$ within $10 \mathrm{ppm}$ of the authentic standard, and the correct fragmentation spectrum (MS/MS) to the standard. The MS/MS scores are based on a comparison of the ions present in the experimental spectrum to the ions present in the library spectrum. While there may be similarities between these molecules based on one of these factors, the use of all three data points can be utilized to distinguish and differentiate biochemicals. More than 3300 commercially available purified standard compounds have been acquired and registered into laboratory information management system (LIMS) for analysis on all platforms for determination of their analytical characteristics. Metabolon data analysts use proprietary visualization and interpretation software to confirm the consistency of peak identification among the various samples. Library matches for each compound were checked for each sample and corrected if necessary. Metabolon classified known metabolites by "super-pathway," representing chemical classes, and "sub-pathway," corresponding to the specific role of a compound in metabolism, on the basis of the Kyoto Encyclopedia of Genes and Genomes (KEGG) pathways. $^{20}$

\section{7 $\quad$ Statistical analysis of metabolomics data}

Metabolomics data were log-transformed then z-score normalized. Linear regression models for association analysis were run using the R statistical package (version 2.14, www.r-project.org/). A model incorporating $\mathrm{CD}$ as a categorical variable with two levels (low/moderate \& high) was used after correcting for covariates including gender, hemolysis levels (determined visually by Metabolon) and ethnic groups. A stringent Bonferroni level of significance of $P<=0.05 / 751=6.6 \times 10^{-5}$ was used to infer association. Orthogonal partial least square discriminant analysis (OPLS-DA) was used to detect components that best distinguish between predefined groups of samples while separating the orthogonal components which do not differentiate between these groups. In this study, OPLS-DA was used to compare elite athletes with low/moderate vs high CD. Both principal component analysis (PCA) and OPLS-DA were run using SIMCA 14 with a threshold percentage of missing metabolite values of $<50 \%$ (a default metabolite-wise metabolite missingness threshold). Gaussian graphical modeling (GGM) was used to identify correlated metabolites, leading potentially to unbiased reconstruction of metabolic reactions as previously reported. ${ }^{21}$ Heatmap analysis was performed using 34 metabolites representing classes that showed most significant differences by $\mathrm{CD}$.

\section{3 | RESULTS}

\section{1 | 112 metabolites significantly differentiate CD groups}

Non-targeted metabolomics was applied to compare the metabolic signature of elite athletes with low/moderate versus high $\mathrm{CD}$. Linear regression model was used to analyze 751 known metabolites (Supplemental Table S1), among which 112 were found significantly different between the two groups $\left(P<6.6 \times 10^{-5}\right)$, including 57 lipids, 25 amino acids, 12 peptides, 8 xenobiotics, 5 nucleotides, 3 carbohydrates, and 2 cofactors and vitamins (Table S1). Sub-pathways describing the predominant changes between the two CD groups included diacylglycerols, fatty acid metabolism (acyl carnitine), eicosanoids, gamma-glutamyl amino acid, lysophospholipid, and phospholipids. Other significant changes were also observed in two aminosugars, metabolites of various amino acids (glycine, serine, threonine, histidine, leucine, isoleucine, valine, methionine, cysteine, and tyrosine), progestin steroids, plasmalogens, purine metabolites, urea cycle metabolites, vitamin A, and xanthine metabolites (Table S1).

Thirty-two metabolites representing different subpathways that significantly changed between the two CD groups were projected on the heatmap in Figure 1. The heatmap gives a snapshot of the metabolites' intensities following correction for the same confounders described earlier in the linear model. Samples were ordered by CD groups into high $\mathrm{CD}$, low/moderate $\mathrm{CD} 1$, and low/moderate CD 2. Whereas the former two groups showed similar intensities of metabolites, the latter group was clearly different, suggesting a presence of a group of athletes with the low/moderate $\mathrm{CD}$ group exhibiting a similar metabolic profile to the high CD group than their own low/moderate CD group.

An OPLS-DA comparing low/moderate versus high CD classes was performed. The statistical model explained variance 
F I G URE 1 Heatmap of metabolites that were significantly associated with cardiovascular demand (CD) from the linear model association analysis (x-axis). Samples on $y$-axis were ordered by sport group (high $\mathrm{CD}$, low/moderate $\mathrm{CD} 1$, and low/moderate CD2). The color code denotes $z$-scaled values of metabolites after correction of confounders (red represents an increase in high $\mathrm{CD}$, and green represents a decrease in high CD)

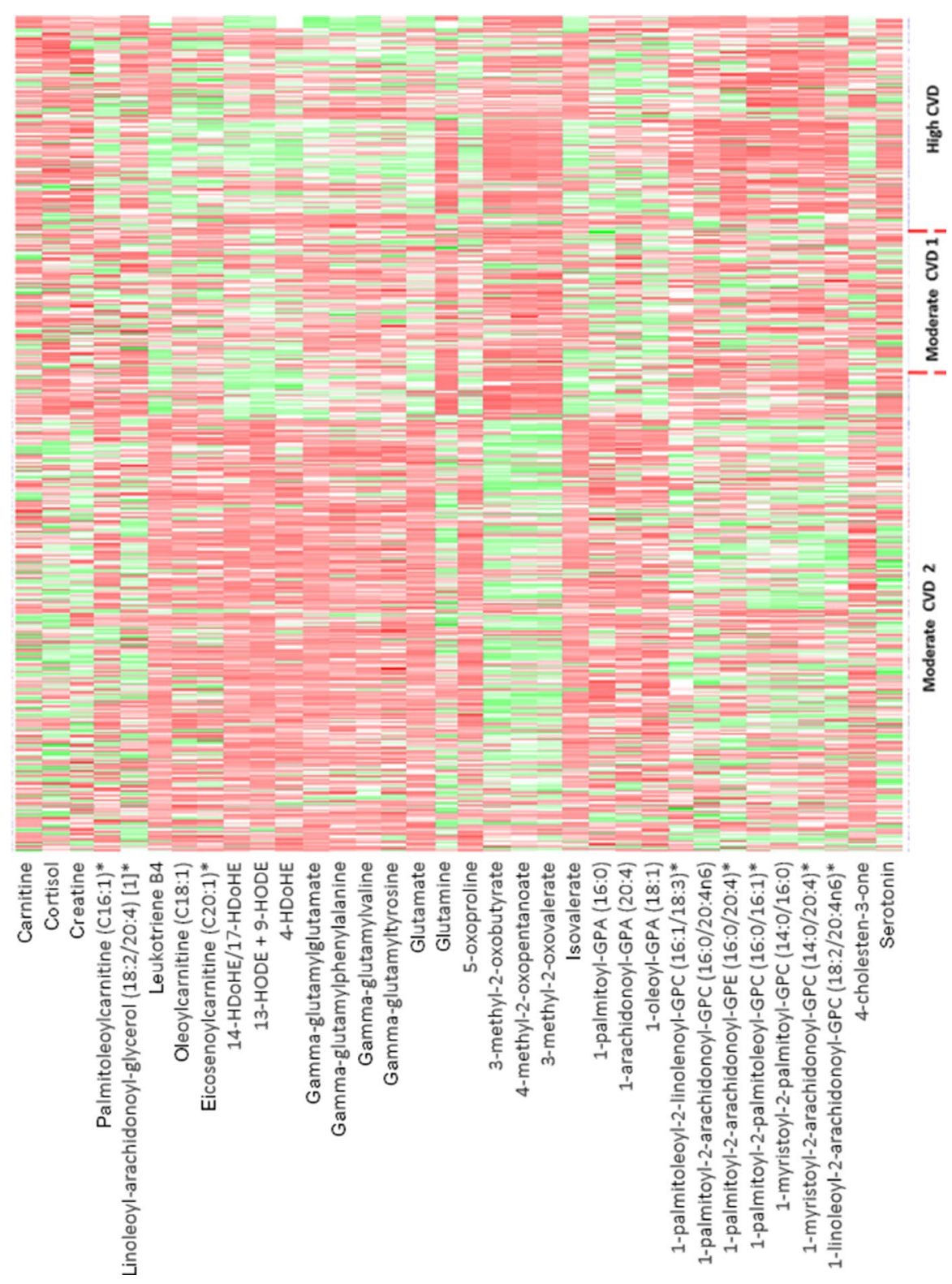

and predictability well. The diagnostic performance computed via the leave-one-out analysis indicated $97.9 \%$ sensitivity and $87.8 \%$ specificity. The model showed one class-discriminatory component accounting for $71 \%$ of the variation in the data due to increased CD (R-squared-Y $=0.71$, Q-squared $=0.52$; Figure 2A). The corresponding loading score, shown in Figure 2B, revealed gamma-glutamyl amino acids, monohydroxy fatty acids, diacylglycerols, leucine, isoleucine and valine metabolites, $\mathrm{PC}$ and $\mathrm{PE}$ as the main metabolites that provide a clear separation between low-medium vs high CD. OPLS, therefore, confirmed linear model results shown in Table S1. Variable importance projection list (VIP list) that reflects the importance of metabolites in the OPLS-DA model with respect to CD groups and the remaining orthogonal component is shown in Table S2. Top metabolites shown in the VIP list also confirm linear model results shown in Table $\mathrm{S} 2$.

\section{2 | Four GGM networks capture pathways that change in relation to $\mathrm{CD}$}

GGM networks were constructed using all 751 metabolites, resulting in 60 subnetworks containing $>2$ metabolites with an overall 600 edges connecting 604 metabolites (nodes) as shown in supplementary Figure S2. The identified subnetworks were filtered for metabolites associated with $\mathrm{CD}$ at $(P<0.05)$, resulting in 11 subnetworks containing $>2$ Bonferroni significant metabolites. Four subnetworks that captured the major metabolic pathways perturbed in relation to $\mathrm{CD}$ were selected for discussion (Figure 3), including carnitine metabolism (Figure 3A), gamma-glutamyl amino acids and their link to glutamate (Figure 3B), cortisol metabolism (Figure 3C), energy metabolites including creatine and TCA (Figure 3D). 

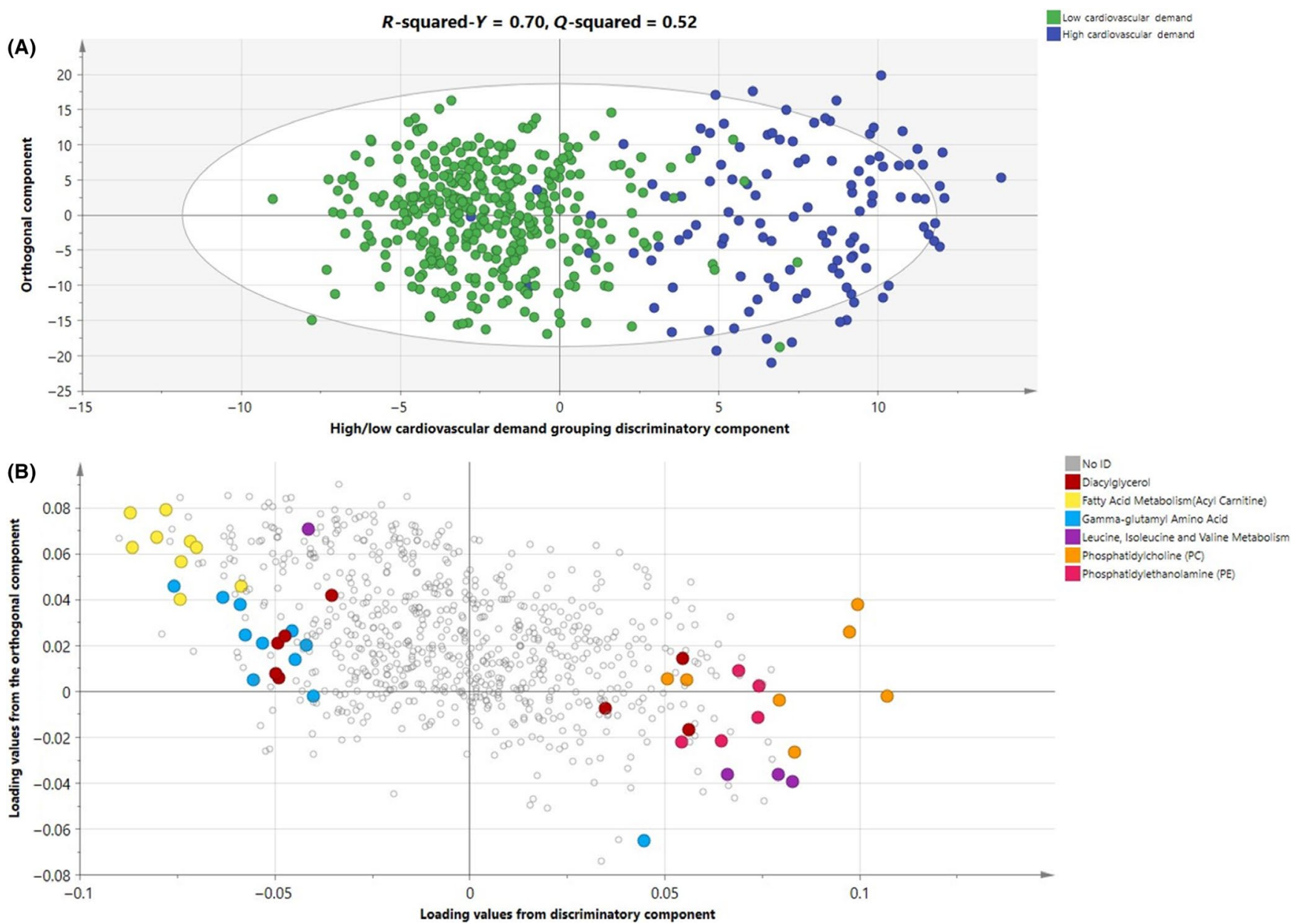

F I G U RE 2 Orthogonal partial least square discriminant analysis model comparing low/moderate vs high cardiovascular demand (CD) classes of elite athletes (A) score plot indicating the class-discriminatory component on the x-axis and the orthogonal component on the y-axis (B) The matching loading plot indicating a cluster of PC and PE at the high end of CD against a cluster of diacylglycerols fatty acids (acyl carnitines) and gamma-glutamyl amino acids at the low/moderate end

\section{4 | DISCUSSION}

The intensive exercise of elite athletes can cause physiological adaptation of the cardiovascular system leading to increased stroke volume and blood pressure in order to enhance performance. Metabolomics profiling of elite athletes with different $\mathrm{CD}$ could provide vital information about their systemic metabolic changes with impact on health and performance. ${ }^{4}$ In this study, a non-targeted metabolic profiling of elite athletes who participated in national or international sport events was performed, followed by comparison of metabolic signatures between athletes who belong to a low/ moderate $\mathrm{CD}$ group versus those who belong to a high $\mathrm{CD}$ group. This study is the first attempt to characterize over 750 metabolites in a large cohort of elite athletes $(n=495)$ with different degrees of $\mathrm{CD}$. The 112 metabolites associated with $\mathrm{CD}$ revealed by the linear regression and confirmed by OPLS-DA were divided into the following two groups: 40 metabolites that showed higher levels in athletes with high
CD compared to their low/moderate CD counterpart and 70 metabolites that showed higher levels in athletes with low/ moderate $\mathrm{CD}$ compared to their high $\mathrm{CD}$ counterpart. The emerging data revealed differences in energy utilization, oxidative stress scavenging mechanisms, and membrane dynamics between the two studied groups. Despite predictability of some of the identified pathways that differentiate high and moderate $\mathrm{CD}$ groups, this study confirms known metabolic mediators of these pathways and identifies novel ones. In particular, the metabolic changes in high $\mathrm{CD}$ group may suggest increased cardiovascular risk, potentially due to exercise-induced left ventricular hypertrophy and increased blood pressure. $^{22}$

Out of the 112 metabolites associated with CD, 40 metabolites showed higher levels in athletes with high $\mathrm{CD}$ compared to their low/moderate CD counterpart. Among the elevated energy-related metabolites in the high $\mathrm{CD}$ group was adenine, the building block of ATP used in cellular metabolism as the main source of energy. Creatine was 


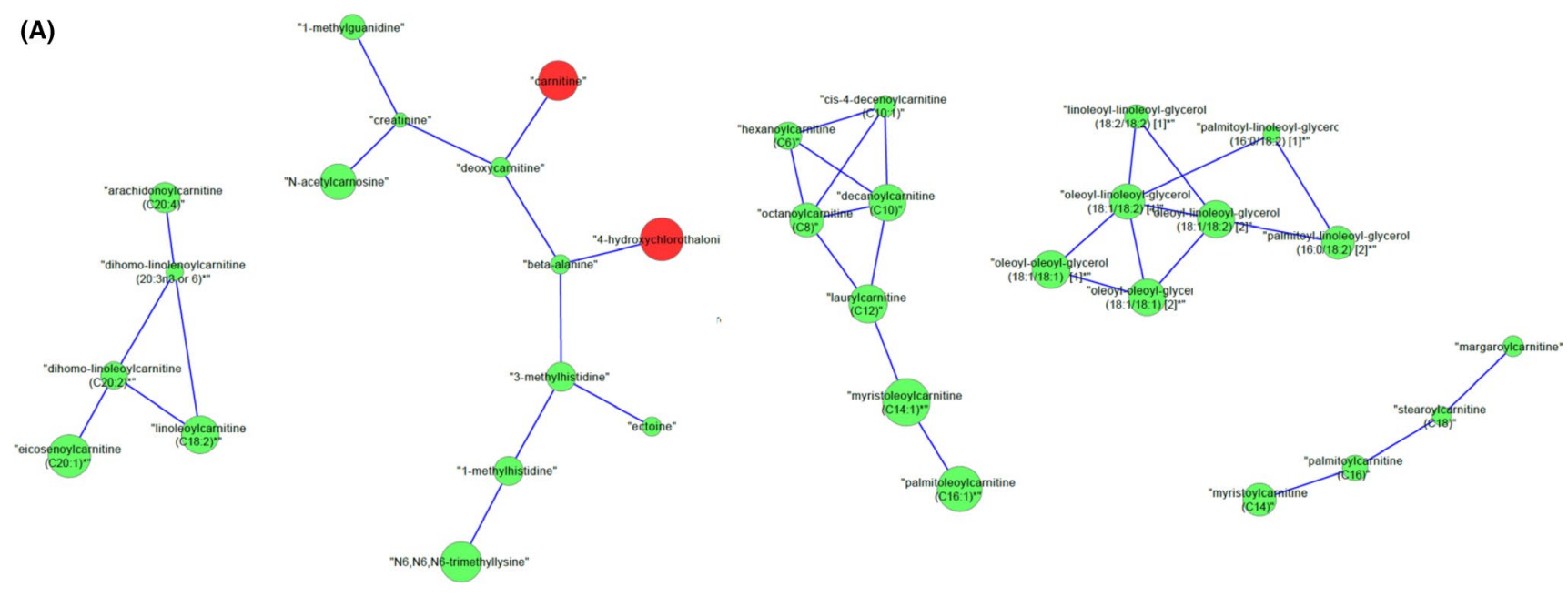

(B)

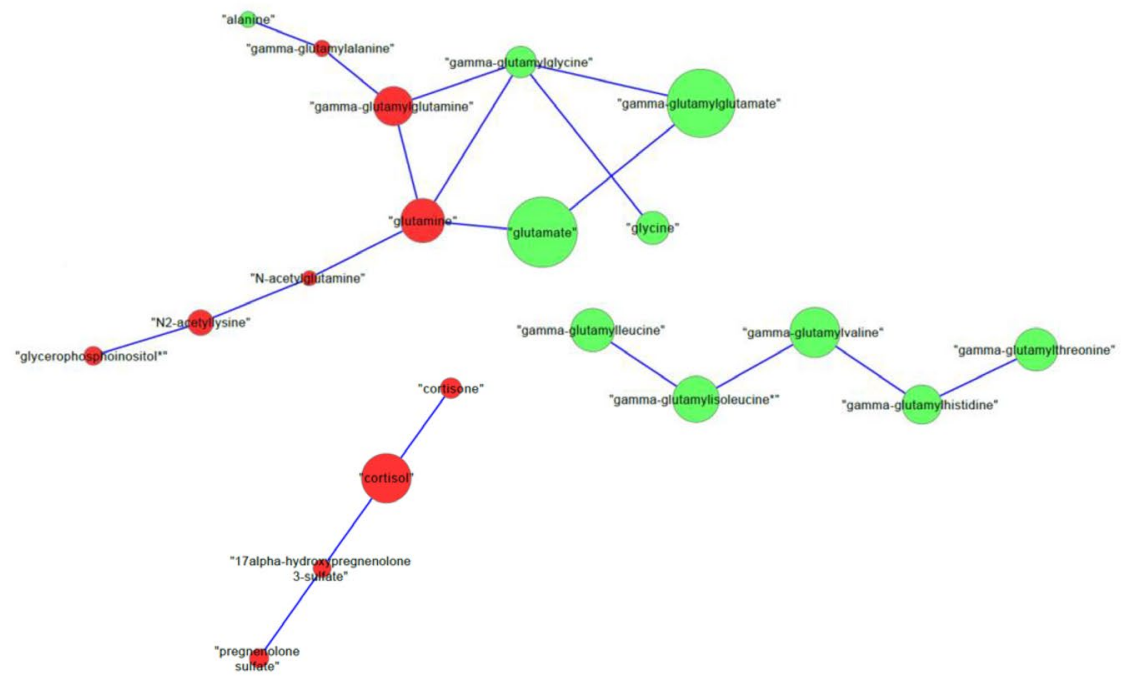

(D)

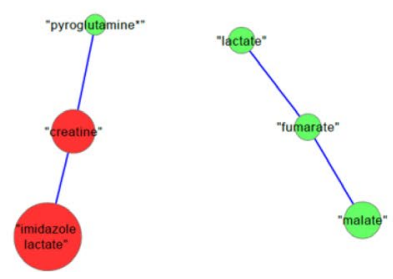

F I G U RE 3 Four Gaussian graphical modelling subnetworks indicate metabolic networks that varied significantly between athletes belonging to low/moderate vs high cardiovascular demand (CD) groups. Changes are represented by nodes with sizes proportional to-(log $P$ value) (larger nodes indicate more significant association with CD group) (A) Carnitine metabolism, (B) gamma-glutamyl amino acids and their link to glutamate, (C) cortisol metabolism, (D) energy metabolites including creatine and tricarboxylic acid cycle. Red color indicates that high CD has higher metabolite levels, and green indicates that low/moderate CD has higher metabolite levels

also increased in the high CD group. It plays a critical role in ATP recycling primarily in muscles via donation of phosphate groups to adenosine diphosphate (ADP). Creatine also acts as a pH buffer in skeletal muscle tissues ${ }^{23}$; therefore, elevated creatine is consistent with the intensive training associated with increased CD. Interestingly, levels of imidazole lactate were positively correlated with levels of creatine as indicated in the GGM subnetworks in Figure
3D, perhaps reflecting increased accumulation of lactate in exercising muscle. Lactate is also used in energy generation through its oxidation to pyruvate by well-oxygenated muscle and heart cells, followed by re-entry into TCA cycle. ${ }^{24}$ Glutamine was also increased in high CD group. It is mostly synthesized in the muscle tissue, accounting for $90 \%$ of all synthesized glutamine, where it can serve as a source of cellular energy next to glucose. ${ }^{25}$ Carnitine was 
also elevated in the high $\mathrm{CD}$ group. Carnitine is known to accumulate in exercising skeletal muscles where it acts as a transporter of long-chain fatty acids into the mitochondria to be oxidized and produce energy. ${ }^{26}$ However, since there was a negative correlation between free carnitine levels and carnitine associated with various fatty acids (Figure 3A), fatty acid oxidation was lower in high $\mathrm{CD}$ group of athletes compared to their low/moderate counterpart. The changes in energy-related metabolites could potentially be related to the long-lasting effect of vigorous exercise in the high $\mathrm{CD}$ group as previously shown. ${ }^{27}$

In addition to energy-related metabolites, specific signaling molecules were increased in high CD group. Among these, three different diacylglycerols containing arachidonic acid (C20:4) were elevated in athletes with high CD including linoleoyl-arachidonoyl-glycerol, palmitoyl-arachidonoyl-glycerol, and palmitoleoyl-arachidonoyl-glycerol. Skeletal muscle is an active site of arachidonic acid retention, accounting for $10 \%-20 \%$ of the phospholipid fatty acid content on average. ${ }^{28}$ In addition to its important function as a second messenger involved in regulating various signaling enzymes from the phospholipase C family (PLC- $\gamma$, PLC- $\delta$, and PKC- $\alpha / \beta / \gamma)$, arachidonic acid plays a critical role as a primary inflammatory intermediate. ${ }^{28}$

Plasmologens were also found to be increased in high CD athletes compared to low/moderate counterparts. This group of metabolites represent up to $20 \%$ of the total phospholipid mass in humans and $\geq 50 \%$ of the ethanol amines fraction in the brain, heart, neutrophils, and eosinophils. ${ }^{29}$ They play key roles as signaling molecules and as modulators of membrane dynamics, providing unique structural properties, mediating various signaling processes, and guarding membrane lipids from oxidation. ${ }^{29}$ GGM subnetworks revealed positive correlations among various plasmalogens, GPEs and GPCs (Figure S2), suggesting biochemical relationships among these various phosphatides.

Cortisol was also increased in high CD. It belongs to the glucocorticoid class of hormones released in response to stress and low blood glucose. It plays important roles in stimulating gluconeogenesis to increase blood sugar, ${ }^{30}$ immunomodulation, and metabolism of fat, protein, and carbohydrates. ${ }^{31}$ Cortisol is also essential for maintenance of normal blood pressure and in excess can cause hypertension. ${ }^{32}$ Therefore, the elevated cortisol in high $\mathrm{CD}$ athletes could reflect their response to increased stress and low blood glucose, potentially causing increased blood pressure in this group of athletes. Interestingly, GGM subnetwork analysis (Figure 3C) also revealed a positive correlation between cortisol and other steroids including pregnenolone, an intermediate in the biosynthesis of most of the steroid hormones, suggesting increased steroid biosynthesis shown previously in endurance athletes. ${ }^{19}$

An increase in branched-chain amino acid (BCAAs, leucine, isoleucine, and valine) metabolites was also evident in the high CD group of athletes as manifested by elevated levels of 3-methyl-2-oxovalerate, 3-methyl-2-oxobutyrate, and 4-methyl-2-oxopentanoate. Exercise is known to promote energy expenditure and stimulates oxidation of BCAAs that play an important role as substrates to TCA intermediates and gluconeogenesis. ${ }^{33}$ Leucine also plays a role as a regulator of intracellular signaling pathway, promoting muscle-protein synthesis in vivo. ${ }^{34}$

Although exercise is generally known to improve wellbeing, it can also increase the risk of heart disease including arrhythmias, myocardial infarction, aortic dissection, and sudden cardiac arrest. ${ }^{35}$ Elevations in several metabolites associated with high CD including BCAAs, PC, and $\mathrm{PE}$ may also suggest an increased risk of cardiovascular disease among athletes who belong to this group. Whereas BCAAs were previously correlated with increased risk of cardiovascular disease, ${ }^{36} \mathrm{PC}$ were shown to be associated with increased cardiovascular mortality independent of traditional risk factors. ${ }^{37} \mathrm{PE}$ (precursors of PC) have also been shown previously to be among the strongest predictive lipid species for risk of cardiovascular disease. ${ }^{38}$ Whether these associations are truly predictive of increased risk of cardiovascular disease or just a reflection of differences in exercise and dietary requirement in high $\mathrm{CD}$ athletes compared to their low/moderate counterpart remains to be investigated. However, it is plausible to assume that high $C D$ athletes could manifest markers of higher risk of cardiovascular disease due to their intensive physical training that is associated with electrical, structural, and functional myocardial adaptations, ${ }^{2,3}$ causing left ventricular hypertrophy and increased blood pressure. ${ }^{22}$

Among the 112 metabolites associated with CD, 70 metabolites showed higher levels in athletes belonging to the low/moderate $\mathrm{CD}$ group compared to their counterparts in the high CD group, including metabolites indicative of energy generation, oxidative stress, and sterol biosynthesis. Elevation in a number of DAGs, fatty acid-carnitines and acylated carnitines suggest enhanced hydrolysis of diacylglycerols, followed by transfer of fatty acids inside the cells for oxidation and energy production. ${ }^{39}$ These changes suggest that low/moderate $\mathrm{CD}$ athletes exhibit a higher beta-oxidation of fatty acids for energy generation, thus a greater potential to activate lipolysis during exercise than their counterpart in the high CD group. Additionally, accumulation of acylated carnitine may reflect a superior exercise recovery than athletes in the high $\mathrm{CD}$ group as carnitine can lower plasma lactate and prolonged exhaustion. ${ }^{40}$ Interestingly, four DAGs containing oleic acid, C18:1 (arachidonic acid precursor), were increased in this group (Table S1). The hypotensive effect of oleic acid may partially explain its increase in this group that is characterized by lower blood pressure. ${ }^{41}$ Additionally, eicosanoids (products of arachidonic acid) were also increased in athletes with low/moderate CD including 
5 and 12 HETE and leukotriene B4 and B5. Levels of these eicosanoids were positively correlated as revealed by Figure 3B. Eicosanoids play a crucial role in various physiological and pathological processes such as immunomodulation and platelet aggregation. ${ }^{42,43}$

Other metabolites were higher in low CD group including glutamate, beta-citrylglutamate, gamma-glutamyl amino acids, and 5-oxoproline, a metabolite of glutathione cycle, suggesting enhanced anti-oxidative stress scavenging mechanism in this group of athletes. ${ }^{44}$ Indeed, GGM subnetworks showed a strong positive correlation between glutamate, gamma-glutamylglutamate, and various gamma-glutamyl amino acids (Figure 3D), suggesting a biochemical and functional relationship.

\section{1 | Study limitations}

Some limitations in the study design were encountered. A batch effect related to sample collection from multiple sites was unavoidable. Sources of batch effect include process and time (IN or OUT of competition) of blood collection, fasting or non-fasting blood status, duration of transportation and storage, potentially influencing metabolic profiling of collected samples. ${ }^{45,46}$ Time since last exercise was a particular concern since its metabolic signature was detected in our previous pilot data. ${ }^{19}$ In spite of these potential confounders, there were no obvious principal components revealed by the PCA of metabolites (Figure S1) that may have accounted for their presence although their potential confounding effects cannot be ruled out. Indeed, controlling collection, storage, and handling of samples is a key component of any metabolomics experimental pipeline. The lack of which could pose major confounders to the data analyses. Although the PCA plot did not reveal obvious confounders, potentially due to noise or highly correlated features driving the separation between the CVD groups, it is possible that the observed differences revealed by OPLS-DA predictive modeling reflect differences in sample handling or storage. Nevertheless, significant metabolic signatures were found following correction for known confounding factors such as hemolysis level and ethnicity. The limited information about participating athletes such as their age, BMI, dietary intake, and training regiments has too potentially reduced the power to identify associated metabolites. However, the generally young age of elite athletes in addition to their diverse sport disciplines may have reduced the impact of their confounding effects. Additionally, the lack of information related to athletes who belong to team sports with respect to their roles was another limitation. In soccer, for example, defenders, mid-fielders, and strikers vary in their cardiovascular demand due to their intensity of exercise and style of playing such as change of direction known to elicit greater metabolic and cardiovascular demands compared to straight line running at the same mean speed. ${ }^{47,48}$ This may explain the presence of a low/moderate $\mathrm{CD}$ group (moderate $\mathrm{CD} 1$ ) that shows similar metabolic pattern to the high CD group in the heatmap (Figure 1). This group is mainly composed of soccer players, some of whom potentially exhibit similar exercise patterns and playing style to those who belong to high CD group. Finally, variations in dietary intake between the studied groups such as supplements, medications, and other ergogenics may have affected their metabolic profile. ${ }^{49,50}$ These differences are challenging to account for as they vary among athletes and are not usually disclosed. Taking all these limitations on board, conclusions on differences between CVD groups are somewhat speculative and require further confirmation and validation in other sport groups.

In summary, findings of this study present a snapshot summarizing differences between two groups of elite athletes with different cardiovascular demand. These metabolic differences were mostly related to source of energy, mechanisms for scavenging oxidative stress, and membrane dynamics. The data also suggest an increased cardiovascular risk in the high CD group compared to their low/moderate counterpart, despite the possibility that these metabolic changes may reflect differences in other environmental factors between the two groups such as diet and exercise. Replication studies are needed to confirm these metabolic differences in independent data set, aiming for discovery of biomarkers for assessing health, performance, and recovery of elite athletes. Such biomarkers could be used as early signs of extreme trainability associated with performance of elite athletes with possible implementations in guiding training programs, avoiding potential disorders related to intensive training, and improving the overall performance of elite athletes.

\section{2 $\quad$ Perspective}

In sport physiology, the intensive exercise of athletes can lead to physiological adaptations in the cardiovascular system to maintain efficient blood flow in exercising muscles. ${ }^{2-4}$ These changes include increasing the stroke volume and blood pressure, known collectively as cardiovascular demand. Although exercise-triggered cardiac hypertrophy and increased blood pressure share common features with cardiovascular disease, ${ }^{22}$ exercise has mostly been regarded to be beneficial for cardiovascular health. ${ }^{51}$

The emerging evidence shows a unique blood metabolic signature of elite athletes with high and low-moderate cardiovascular demand. The metabolic signature associated with high cardiovascular demand indicates accumulation of certain metabolic markers of cardiovascular disease such as diacylglycerides containing arachidonic acid, branchedchain amino acids, plasminogens, phosphatidylcholines, and phosphatidylethanolamines. Whether these metabolites are true indicators of higher cardiovascular risk or merely a 
consequence of cardiac hypertrophy or other environmental factors such as diet and exercise remains to be investigated. Monitoring cardiac function in elite athletes with high cardiovascular demand may therefore have the potential to reduce their risk of cardiovascular disease.

\section{ACKNOWLEDGEMENTS}

This study was funded by Qatar National Research Fund (QNRF), Grant number NPRP7-272-1-041 (MAE, KS, CG and $\mathrm{FB}$ ).

\section{CONFLICT OF INTEREST}

The authors declare that they have no competing interests.

\section{AUTHOR CONTRIBUTIONS}

FK, FD, FB, AL, DA, AH, CG, KS, NY, and MAE collected samples, carried out analysis, wrote the paper, reviewed, and accepted its final version. MAE (corresponding) is responsible for the integrity of the work as a whole.

\section{ETHICS APPROVAL AND CONSENT TO PARTICIPATE}

This study was performed in accordance with the World Medical Association Declaration of Helsinki. All protocols were approved by the Institutional Research Board of anti-doping lab Qatar (F2014000009), and participants have given consent to participate.

\section{DATA AVAILABLITY}

The data sets used and/or analyzed during the current study are available from the corresponding author on reasonable request.

\section{ORCID}

Mohamed A. Elrayess (D) https://orcid. org/0000-0003-3803-4604

\section{REFERENCES}

1. Swann C, Moran A, Piggott D. Defining elite athletes: issues in the study of expert performance in sport psychology. Psychol Sport Exerc. 2015;16(1):3-14.

2. Chevalier L, Kervio G, Corneloup L, et al. Athlete's heart patterns in elite rugby players: effects of training specificities. Arch Cardiovasc Dis. 2013;106(2):72-78.
3. Barbier J, Ville N, Kervio G, Walther G, Carre F. Sports-specific features of athlete's heart and their relation to echocardiographic parameters. Herz. 2006;31(6):531-543.

4. Gonzalez-Alonso J. Human thermoregulation and the cardiovascular system. Exp Physiol. 2012;97(3):340-346.

5. Rowell LB. Human Cardiovascular Control. New York, NY: Oxford University Press; 1993.

6. Laughlin MH. Cardiovascular response to exercise. Am J Physiol. 1999;277(6 Pt 2):S244-259.

7. Mitchell JH, Haskell W, Snell P, Van Camp SP. Task force 8: classification of sports. J Am Coll Cardiol. 2005;45(8):1364-1367.

8. Fagard R. Athlete's heart. Heart. 2003;89(12):1455-1461.

9. Zivkovic AM, German JB. Metabolomics for assessment of nutritional status. Curr Opin Clin Nutr Metab Care. 2009;12(5):501-507.

10. Yan B, A J, Wang G, et al. Metabolomic investigation into variation of endogenous metabolites in professional athletes subject to strength-endurance training. J Appl Physiol. 2009;106(2):531-538.

11. Nicholson JK, Connelly J, Lindon JC, Holmes E. Metabonomics: a platform for studying drug toxicity and gene function. Nat Rev Drug Discov. 2002;1(2):153-161.

12. Heaney LM, Deighton K, Suzuki T. Non-targeted metabolomics in sport and exercise science. J Sports Sci. 2019;37(9):959-967.

13. Lewis GD, Farrell L, Wood MJ, et al. Metabolic signatures of exercise in human plasma. Sci Transl Med. 2010;2(33):33ra37.

14. Goodwin ML, Harris JE, Hernandez A, Gladden LB. Blood lactate measurements and analysis during exercise: a guide for clinicians. J Diabetes Sci Technol. 2007;1(4):558-569.

15. Berton R, Conceição MS, Libardi CA, et al. Metabolic time-course response after resistance exercise: a metabolomics approach. $J$ Sports Sci. 2017;35(12):1211-1218.

16. Dudzinska W, Lubkowska A, Dolegowska B, Safranow K, Jakubowska K. Adenine, guanine and pyridine nucleotides in blood during physical exercise and restitution in healthy subjects. Eur J Appl Physiol. 2010;110(6):1155-1162.

17. Howarth KR, LeBlanc PJ, Heigenhauser GJ, Gibala MJ. Effect of endurance training on muscle TCA cycle metabolism during exercise in humans. J Appl Physiol. 2004;97(2):579-584.

18. Peake JM, Tan SJ, Markworth JF, Broadbent JA, Skinner TL, Cameron-Smith D. Metabolic and hormonal responses to isoenergetic high-intensity interval exercise and continuous moderate-intensity exercise. Am J Physiol Endocrinol Metab. 2014;307(7):E539-552

19. Al-Khelaifi F, Diboun I, Donati F, et al. A pilot study comparing the metabolic profiles of elite-level athletes from different sporting disciplines. Sports Med Open. 2018;4(1):2.

20. Kanehisa M, Goto S. KEGG: kyoto encyclopedia of genes and genomes. Nucleic Acids Res. 2000;28(1):27-30.

21. Krumsiek J, Suhre K, Illig T, Adamski J, Theis FJ. Gaussian graphical modeling reconstructs pathway reactions from high-throughput metabolomics data. BMC Syst Biol. 2011;5:21.

22. Lovic D, Narayan P, Pittaras A, Faselis C, Doumas M, Kokkinos P. Left ventricular hypertrophy in athletes and hypertensive patients. J Clin Hypertens (Greenwich). 2017;19(4):413-417.

23. Wallimann T, Tokarska-Schlattner M, Schlattner U. The creatine kinase system and pleiotropic effects of creatine. Amino Acids. 2011;40(5):1271-1296.

24. Cruz RSdO, de Aguiar RA, Turnes T, Penteado Dos Santos R, Fernandes Mendes de Oliveira M, Caputo F. Intracellular 
shuttle: the lactate aerobic metabolism. ScientificWorldJournal. 2012;2012:420984.

25. Aledo JC. Glutamine breakdown in rapidly dividing cells: waste or investment? BioEssays. 2004;26(7):778-785.

26. Roepstorff C, Vistisen B, Roepstorff K, Kiens B. Regulation of plasma long-chain fatty acid oxidation in relation to uptake in human skeletal muscle during exercise. Am J Physiol Endocrinol Metab. 2004;287(4):E696-705.

27. Knab AM, Shanely RA, Corbin KD, Jin F, Sha W, Nieman DC. A 45-minute vigorous exercise bout increases metabolic rate for 14 hours. Med Sci Sports Exerc. 2011;43(9):1643-1648.

28. Korotkova M, Lundberg IE. The skeletal muscle arachidonic acid cascade in health and inflammatory disease. Nat Rev Rheumatol. 2014;10(5):295-303.

29. Braverman NE, Moser AB. Functions of plasmalogen lipids in health and disease. Biochem Biophys Acta. 2012;1822(9):1442-1452.

30. Khani S, Tayek JA. Cortisol increases gluconeogenesis in humans: its role in the metabolic syndrome. Clin Sci (Lond). 2001;101(6):739-747.

31. Christiansen JJ, Djurhuus CB, Gravholt CH, et al. Effects of cortisol on carbohydrate, lipid, and protein metabolism: studies of acute cortisol withdrawal in adrenocortical failure. J Clin Endocrinol Metab. 2007;92(9):3553-3559.

32. Kelly JJ, Mangos G, Williamson PM, Whitworth JA. Cortisol and hypertension. Clin Exp Pharmacol Physiol Suppl. 1998;25:S51-56.

33. Shimomura Y, Murakami T, Nakai N, Nagasaki M, Harris RA. Exercise promotes BCAA catabolism: effects of BCAA supplementation on skeletal muscle during exercise. J Nutr. 2004;134(6 Suppl):1583S-1587S.

34. Wolfe RR. Branched-chain amino acids and muscle protein synthesis in humans: myth or reality? J Int Soc Sports Nutr. 2017;14:30.

35. Lawless CE, Olshansky B, Washington RL, et al. Sports and exercise cardiology in the United States: cardiovascular specialists as members of the athlete healthcare team. J Am Coll Cardiol. 2014;63(15):1461-1472.

36. Tobias DK, Lawler PR, Harada PH, et al. Circulating branchedchain amino acids and incident cardiovascular disease in a prospective cohort of US women. Circ Genom Precis Med. 2018;11(4):e002157.

37. Zheng Y, Li Y, Rimm EB, et al. Dietary phosphatidylcholine and risk of all-cause and cardiovascular-specific mortality among US women and men. Am J Clin Nutr. 2016;104(1):173-180.

38. Stegemann C, Pechlaner R, Willeit P, et al. Lipidomics profiling and risk of cardiovascular disease in the prospective populationbased Bruneck study. Circulation. 2014;129(18):1821-1831.

39. Hoppel CL. Carnitine and carnitine palmitoyltransferase in fatty acid oxidation and ketosis. Fed Proc. 1982;41(12):2853-2857.
40. Orer GE, Guzel NA. The effects of acute L-carnitine supplementation on endurance performance of athletes. J Strength Cond Res. 2014;28(2):514-519.

41. Teres S, Barcelo-Coblijn G, Benet M, et al. Oleic acid content is responsible for the reduction in blood pressure induced by olive oil. Proc Natl Acad Sci U S A. 2008;105(37):13811-13816.

42. Lone AM, Tasken K. Proinflammatory and immunoregulatory roles of eicosanoids in T cells. Front Immunol. 2013;4:130.

43. Dennis EA, Norris PC. Eicosanoid storm in infection and inflammation. Nat Rev Immunol. 2015;15(8):511-523.

44. Liu Y, Hyde AS, Simpson MA, Barycki JJ. Emerging regulatory paradigms in glutathione metabolism. Adv Cancer Res. 2014;122:69-101.

45. Yin $\mathrm{P}$, Lehmann $\mathrm{R}, \mathrm{Xu}$ G. Effects of pre-analytical processes on blood samples used in metabolomics studies. Anal Bioanal Chem. 2015;407(17):4879-4892.

46. Naz S, Vallejo M, Garcia A, Barbas C. Method validation strategies involved in non-targeted metabolomics. J Chromatogr A. 2014;1353:99-105.

47. Buchheit M, Haydar B, Hader K, Ufland P, Ahmaidi S. Assessing running economy during field running with changes of direction: application to $20 \mathrm{~m}$ shuttle runs. Int J Sports Physiol Perform. 2011;6(3):380-395.

48. Buchheit M, Laursen PB. High-intensity interval training, solutions to the programming puzzle. Part II: anaerobic energy, neuromuscular load and practical applications. Sports Med. 2013;43(10):927-954.

49. Williams MH. Dietary supplements and sports performance: introduction and vitamins. J Int Soc Sports Nutr. 2004;1:1-6.

50. Al-Khelaifi F, Diboun I, Donati F, et al. Metabolomics profiling of xenobiotics in elite athletes: relevance to supplement consumption. J Int Soc Sports Nutr. 2018;15(1):48.

51. Sharma S, Merghani A, Mont L. Exercise and the heart: the good, the bad, and the ugly. Eur Heart J. 2015;36(23):1445-1453.

\section{SUPPORTING INFORMATION}

Additional supporting information may be found online in the Supporting Information section at the end of the article.

How to cite this article: Al-Khelaifi F, Donati F, Botrè $\mathrm{F}$, et al. Metabolic profiling of elite athletes with different cardiovascular demand. Scand J Med Sci Sports. 2019;29:933-943. https://doi.org/10.1111/ $\underline{\text { sms. } 13425}$ 